\title{
FLUCTUATIONS ET CORRÉlations DE PHASE DANS LES SUPRACONDUCTEURS GRANULAIRES COUPLÉS PAR EFFET JOSEPHSON
}

\author{
P. PELLAN, G. DOUSSELIN \\ INSA, 35031 Rennes, France \\ et \\ J. ROSENBLATT
}

INSA, 35031 Rennes et CNET, 22000 Lannion, France

\begin{abstract}
Résumé. - Nous étudions les propriétés statistiques des contacts Josephson dues aux fluctuations thermodynamiques de la différence des phases supraconductrices. Cette analyse est généralisée aux ensembles tri- et bidimensionnels de jonctions, dont les résultats expérimentaux mettent en évidence une transition de phase entre un état paracohérent et un état cohérent, où les phases des supraconducteurs sont ordonnées.

Abstract. - Statistical properties of Josephson contacts, derived from assumed thermodynamic fluctuations of the superconducting phase difference, are examined. Generalisation of this analysis to three- and two-dimensional assemblies of junctions, together with experimental evidence, show a phase transition from a paracoherent to a coherent state, where phases in the superconductors achieve ordering.
\end{abstract}

1. Introduction. - Il est bien connu que l'effet Josephson établit entre deux supraconducteurs liés par une jonction, une énergie de couplage

$$
E=\frac{\Phi_{0}}{2 \pi} I_{\mathrm{S}} \cos \varphi=J_{\mathrm{S}} \cos \varphi
$$

où $\Phi_{0}$ est le quantum de flux, $I_{\mathrm{S}}$ le supercourant maximum de la jonction et $\varphi$ la différence des phases du paramètre d'ordre $\Delta(T)$ dans les deux supraconducteurs [1], [2]. En présence d'un courant appliqué $I$ ce couplage donne une contribution à l'énergie libre du système [2]

$$
F_{J}=-J_{\mathrm{S}} \cos \varphi-J \varphi
$$

où $J$ est défini par $J=\Phi_{0} I / 2 \pi$. Près de la température critique, le paramètre $J_{\mathrm{S}}$ est proportionnel à $|\Delta|^{2} / k T \propto T_{\mathrm{c}}-T$. D'autre part, l'énergie libre de condensation supraconductrice $F_{\mathrm{S}}$ est proportionnelle au volume des électrodes et ne dépend pas de la phase. Il peut exister un domaine de températures où

$$
\left|F_{\mathrm{S}}\right| \gg k T \gtrsim E,
$$

c'est-à-dire où $F_{\mathrm{S}}$ et par conséquent $|\Delta(T)|$ ont leurs valeurs d'équilibre tandis que $E$ peut subir des variations autour d'une valeur moyenne par l'intermédiaire des variations de la différence de phase $\varphi$. Plus précisément, la condition pour l'absence de fluctuations $\operatorname{de}|\Delta(T)|$ à $T<T_{\mathrm{c}}$ est que toutes les dimensions linéaires des électrodes soient plus grandes que la longueur de cohérence $\xi(T)$, ce que nous supposons par la suite.

Nous étudierons successivement la formulation statistique du problème dans le cas d'une jonction ainsi que sa généralisation au cas d'une chaîne linéaire et les résultats théoriques et expérimentaux concernant des ensembles tri- et bidimensionnels de jonctions.

2. Description statistique des fluctuations de phase. 2.1 CAS D'UNe SEULE JONCTION. - Le problème posé est celui des fluctuations autour de leur position d'équilibre de deux vecteurs dans le plan complexe, $|\Delta| e^{i \varphi_{1}}$ et $|\Delta| e^{i \varphi_{2}}$, correspondant au paramètre d'ordre dans les électrodes de la jonction. Leur interaction est donnée par l'expression (2), ce qui conduit [3] à la description du système par une fonction partition

$$
Z=\frac{1}{2 \pi} \int_{-\pi}^{\pi} \exp \left(j_{\mathrm{S}} \cos \varphi+j \varphi\right) \mathrm{d} \varphi
$$

où $j=J / k T, j_{\mathrm{S}}=J_{\mathrm{s}} / k T, \varphi=\varphi_{2}-\varphi_{1}$.

La fonction corrélation de ces deux vecteurs est [4] :

$$
C_{12}=\left\langle\mathrm{e}^{i \varphi_{2}} \mathrm{e}^{-i \varphi_{1}}\right\rangle,
$$

avec

$$
<\cos \varphi>=\operatorname{Re} C_{12}=\frac{\partial \ln Z}{\partial j_{\mathrm{s}}}
$$

et l'énergie moyenne de couplage est

$$
\langle E\rangle=J_{\mathrm{S}}<\cos \varphi>\text {. }
$$


La différence de phase moyenne

$$
<\varphi>=\frac{\partial \ln Z}{\partial j}
$$

permet également de définir une susceptibilité de phase

$$
\begin{aligned}
\chi_{\varphi}=\frac{\partial\langle\varphi\rangle}{\partial I} & =\frac{\Phi_{0}}{2 \pi} \beta \frac{\partial^{2} \ln ^{\Gamma} Z}{\partial j^{2}} \\
& =\frac{\Phi_{0}}{2 \pi} \beta\left(<\varphi_{\omega}^{2}>-<\varphi>^{2}\right)
\end{aligned}
$$

où la dernière expression est une conséquence du théorème de fluctuation-dissipation. Signalons qu'en l'absence de fluctuations, où la phase est

$$
\varphi=\arcsin I / I_{\mathrm{s}}, \quad \chi_{\varphi}=\partial \varphi / \partial I=\left(I^{2}-I_{\mathrm{s}}^{2}\right)^{-1 / 2} \rightarrow \infty
$$

pour $I \rightarrow I_{\mathrm{s}}$. Ce comportement est lié à la singularité de la courbe $\mathrm{d} V / \mathrm{d} I$ à $I=I_{\mathrm{s}}$ dans une jonction idéale. On s'attend à voir cette singularité " arrondie » comme conséquence des fluctuations de phase, le maximum correspondant permettant toujours de déterminer $I_{\mathrm{s}}$.

L'éq. (4) pour la fonction partition peut être développée comme suit :

$Z=\frac{\sinh \pi j}{\pi j}\left[I_{0}\left(j_{\mathrm{S}}\right)+2 \sum_{n=1}^{\infty}(-1)^{n} I_{n}\left(j_{\mathrm{s}}\right) \frac{j^{2}}{j^{2}+n^{2}}\right]$

ou $I_{n}\left(j_{\mathrm{S}}\right)$ est la fonction de Bessel modifiée d'ordre $n$. L'expression (9) devient particulièrement simple pour $j \rightarrow 0$. Dans ce cas $Z=I_{0}\left(j_{\mathrm{s}}\right)$ et

$$
<\cos \varphi>_{I=0}=\frac{I_{1}\left(j_{\mathrm{S}}\right)}{I_{0}\left(j_{\mathrm{S}}\right)} .
$$

La figure 1 montre la fonction corrélation et l'énergie de couplage en fonction de $j_{\mathrm{s}}$, et donc de la température. Si l'on tient compte que, pour $T$ proche de $T_{\mathrm{c}}$,

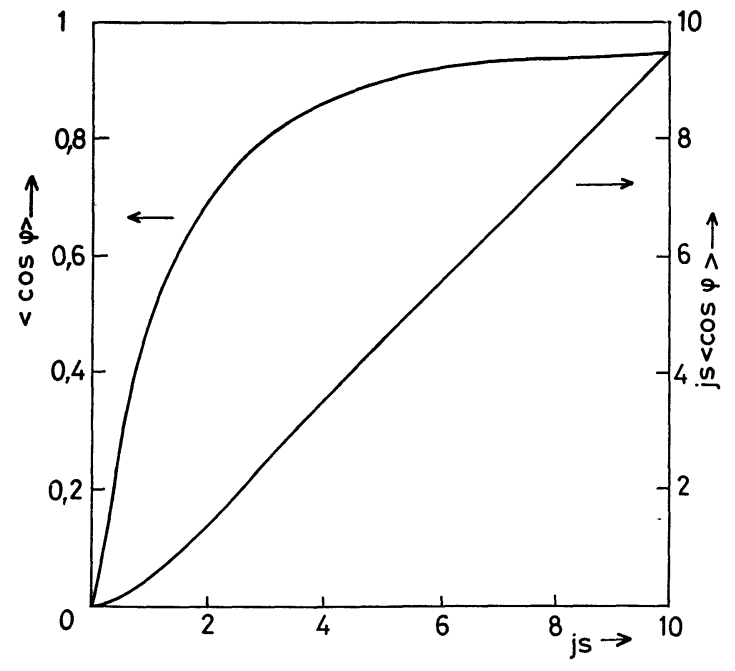

Fig. 1. - Fonction corrélation $\langle\cos \varphi\rangle$ et énergie de couplage à courant appliqué nul en fonction de $j_{\mathrm{s}}$.
$j_{\mathrm{S}}(T) \simeq 4 I_{\mathrm{S}}(0)\left(T_{\mathrm{c}}-T\right)$ quand $I_{\mathrm{S}}$ est exprimé en $\mu \mathrm{A}$ et $T$ en $\mathrm{K}$, on voit que les fluctuations de phase, responsables de la partie non linéaire de la courbe $\beta\langle E\rangle=j_{\mathrm{s}}\langle\cos \varphi\rangle$, devraient être apparentes sur quelques millidegrés seulement en dessous de $T_{\mathrm{c}}$ pour une jonction typique ayant $I_{\mathrm{S}}(0) \simeq 100 \mu \mathrm{A}$.

Nous avons étudié l'effet d'un courant non nul dans le cadre d'un modèle plus simple mathématiquement : l'expression de Josephson pour le supercourant est remplacée par une fonction en dent de scie

$$
J=\frac{J_{\mathrm{S}}(\varphi-2 n \pi)}{\pi}, \quad(n-1) \pi \leqslant \varphi<(n+1) \pi
$$

ce qui est vraisemblablement convenable pour la description des microponts [5]. L'éq. (2) est remplacée par

$$
F_{J}=-J_{\mathrm{S}}\left[\frac{\pi}{2}-\frac{(\varphi-2 n \pi)^{2}}{2 \pi}\right]-J \varphi
$$

On obtient pour l'énergie de couplage

$$
\begin{aligned}
\beta< & E^{t}>=j_{\mathrm{S}} \frac{\partial \ln Z}{\partial j_{\mathrm{S}}}= \\
& =y_{+} y_{-}+\frac{1}{\sqrt{\pi}} \frac{y_{-} \mathrm{e}^{-y_{+}^{2}}+y_{+} \mathrm{e}^{-y_{-}^{2}}}{\operatorname{erf} y_{+}+\operatorname{erf} y_{-}}-\frac{1}{2}
\end{aligned}
$$

où $y_{ \pm}=\sqrt{\pi j_{\mathrm{S}} / 2}(1 \pm \rho)$ et $\rho=j / j_{\mathrm{s}}$ est le rapport entre le courant appliqué et le supercourant maximum. La figure 2 montre les résultats d'un calcul numérique

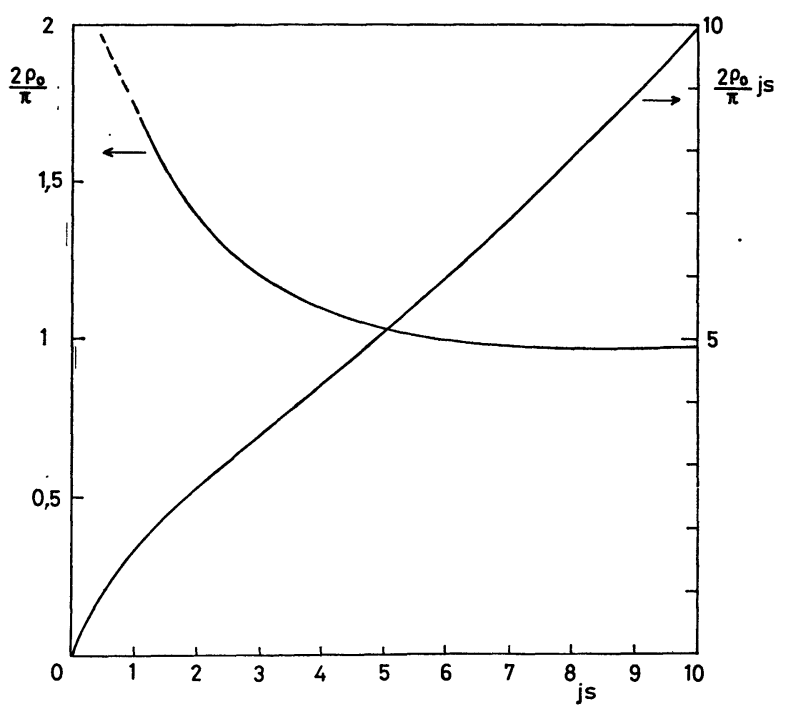

Fig. 2. - Rapport $\rho_{0}$ du courant appliqué au supercourant maximum donnant une réduction de $90 \%$ de l'énergie de couplage pour une jonction caractérisée par une dépendance supercourant-phase en dent de scie.

donnant la valeur $\rho_{0}$ de ce rapport qui réduit $\langle E\rangle$ à $10 \%$ de sa valeur pour $\rho=0$. On voit encore une fois que, même en présence de courant, les effets des fluctuations disparaissent très vite en dessous de $T_{\mathrm{c}}$. 
2.2 Chaîne LiNÉAIRE. - On peut se demander si des systèmes comportant un grand nombre de jonctions seraient plus sensibles aux fluctuations de phase. Effectivement, considérons la fonction partition d'un ensemble de $N$ jonctions branchées en série :

$$
\begin{aligned}
Z=\frac{1}{(2 \pi)^{N}} \int_{-\pi}^{\pi} \ldots \int_{-\pi}^{\pi} \prod_{i=1}^{N} \mathrm{~d} \varphi_{i} \times \\
\quad \times \exp \left[j_{\mathrm{S}} \sum_{i} \cos \varphi_{i}+j \sum_{i} \varphi_{i}\right],
\end{aligned}
$$

où l'on a supposé que toutes les jonctions ont les mêmes propriétés. La fonction partition est séparable, et l'on obtient facilement la fonction corrélation à courant nul entre la première et la dernière jonction [4] :

$$
C_{1 N}=\langle\cos \varphi\rangle^{N}
$$

qui montre que la corrélation est affaiblie à mesure que le nombre de jonctions augmente. Nous comptons publier prochainement des résultats expérimentaux sur de tels systèmes.

3. Supraconducteurs granulaires massifs (6). 3.1 COHÉRENCE ET PARACOHÉRENCE. - Nous avons fabriqué des ensembles de quelques milliers de contacts ponctuels par simple compression de grains de $\mathrm{Nb}$ ou Ta, de diamètre $d \simeq 160 \mu \gg \xi(T)$. En supposant que tous les contacts ont les mêmes propriétés Josephson et que $I=0$, l'expression (2) peut être généralisée immédiatement :

$$
F_{J}=-J_{\mathrm{S}} \sum_{\alpha<\alpha^{\prime}}^{\prime} \cos \varphi_{\alpha \alpha^{\prime}}
$$

où $\varphi_{\alpha \alpha^{\prime}}=\varphi_{\alpha}-\varphi_{\alpha^{\prime}}$ est la différence des phases du paramètre d'ordre dans les grains $\alpha$ et $\alpha^{\prime}$, et la somme est prise sur des premiers voisins seulement. L'analogie avec un ferromagnétique d'Heisenberg avec espace de spin à deux dimensions est immédiate. Cette analogie permet de décrire physiquement le comportement du système. Dans un intervalle de températures

$$
T_{\mathrm{c}} \geqslant T \geqslant T_{0}
$$

les vecteurs représentatifs du paramètre d'ordre dans chaque grain sont orientés au hasard. La valeur moyenne de leur composante réelle est nulle,

$$
<\cos \varphi_{\alpha}>=0 \text {. }
$$

C'est la région paracohérente [7]. A la température de cohérence $T_{0}\left(k T_{0} \simeq p J_{\mathrm{S}}, p\right.$ étant le nombre de premiers voisins) il se produit une transition de phase à un état ordonné où $\left\langle\cos \varphi_{\alpha}\right\rangle \neq 0$. Il paraît naturel de prendre comme paramètre d'ordre de cette transition $m=\left\langle\cos \varphi_{\alpha}\right\rangle$ et d'utiliser une approximation de champ moléculaire. On peut alors montrer [7] que chaque jonction du système se comporte comme si l'intensité du couplage Josephson changeait par la transformation

$$
\left.\left.J_{\mathrm{S}} \rightarrow\langle E\rangle_{I \rightarrow 0}=J_{\mathrm{S}}<\cos \varphi_{\alpha \alpha^{\prime}}\right\rangle+H_{1}<\cos \varphi_{\alpha}\right\rangle,
$$

où $H_{1}$ est le champ moléculaire. Les résultats de ce calcul apparaissent sur la figure 3 , où on a utilisé l'approximation de Bethe [8] pour le champ moléculaire appliquée à un réseau cubique de grains supraconducteurs ayant $T_{0}=0,6 T_{\mathrm{c}}$. Le cas d'une seule

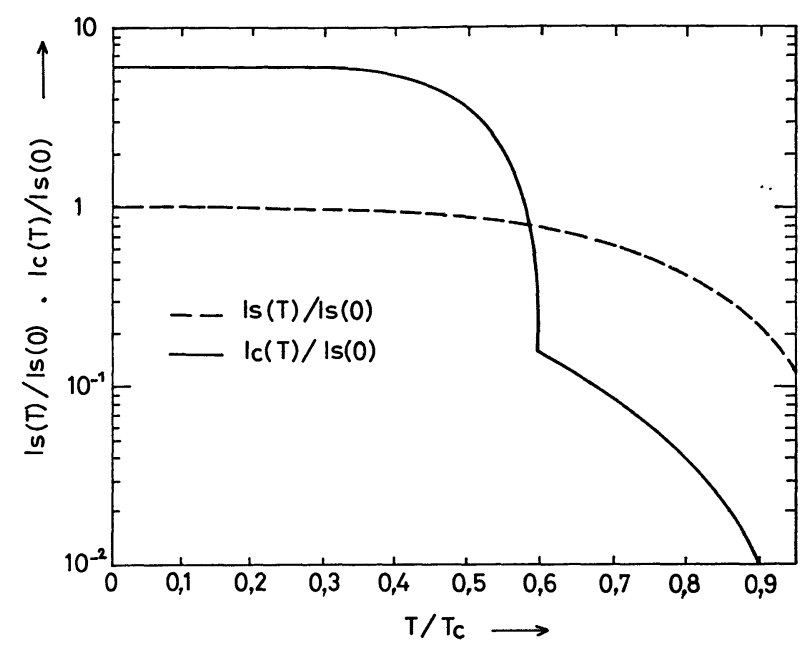

Fig. 3. - Supercourant maximum $I_{\mathrm{S}}(T) / I_{\mathrm{S}}(0)$ en fonction de la température réduite d'après la référence [9] et la même quantité $I_{\mathrm{c}}(T) / I_{\mathrm{S}}(0)$ pour la jonction faisant partie d'un ensemble statistique. La température de cohérence a été prise comme

$$
T_{0}=0,6 T_{\mathrm{c}} \text {. }
$$

jonction en l'absence de fluctuations de phase [9] est montré comme référence. Le supercourant maximum de chaque jonction est affaibli dans la région paracohérente $\left(\left\langle\cos \varphi_{\alpha}\right\rangle=0\right)$ et renforcé dans la région cohérente $\left(T<T_{0}\right)$.

3.2 RÉSULTATS EXPÉRIMENTAUX. - Les calculs précédents peuvent être vérifiés par des mesures de résistance des ensembles granulaires. En effet, comme il a été démontré par ailleurs [10], la présence d'une résistance normale $R_{\mathrm{q}}$ dans la jonction introduit des fluctuations dissipatives qui se traduisent par une résistance non nulle $R$ même à des courants appliqués très faibles. Pour $I \rightarrow 0$ [10] :

$$
\frac{R}{R_{\mathrm{q}}}=\left\{I_{0}\left(\frac{J_{\mathrm{S}}}{k T}\right)\right\}^{-2} .
$$

L'éq. (18) a été obtenue par analogie avec le mouvement brownien d'une particule soumise à une force aléatoire, en supposant que la valeur d'équilibre de la phase est donnée par la condition $\partial F_{J} / \partial \varphi=0$, compte tenu de l'expression (2). Or, nous avons vu que si $\left|F_{J}\right|<k T, \varphi$ est elle-même une quantité qui oscille autour d'une valeur d'équilibre. Dans ce cas, des calculs analogues à ceux de la référence [10] où on introduit les moyennes statistiques de toutes les quantités dépendant de $\varphi$ conduisent naturellement à une équation du type (18) où $J_{\mathrm{s}}$ est remplacé par la transformation (17). Dans le cas d'une seule jonction, évidemment, $H_{1}=0$ et (cf. § 2.1) les effets des fluctuations de phase sont faibles pour des jonctions ayant 
$J_{s}>0,1 \mu \mathrm{A}$. Par contre, ces effets peuvent devenir spectaculaires pour des ensembles granulaires où $H_{1} \neq 0$. La résistance dynamique à courant faible change brutalement en passant par la température de cohérence $T_{0}$. C'est ce que nous montrons sur la figure 4 , où les courbes théoriques ont été obtenues des expressions (17) et (18), avec des moyennes statistiques calculées dans l'approximation de Bethe [8]. Egalement, c'est en dessous de $T_{0}$ qu'on trouve des effets Josephson typiques (tels que la périodicité de la résistance dynamique en fonction du champ magnétique appliqué, par exemple) montrant que le système se comporte comme une "mégajonction "-quand la cohérence de phase se développe.

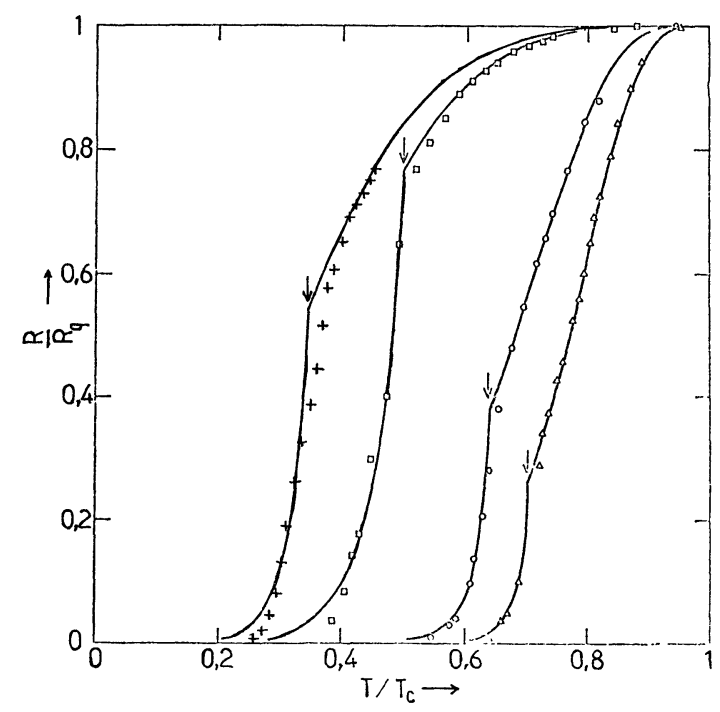

Fig. 4. - Valeurs expérimentales de $R / R_{q}$ pour différents échantillons de $\mathrm{Ta}$ (triangles, cercles et carrés) et de $\mathrm{Nb}$ (croix). Les courbes on̂t été obtenues à partir de l'éq. (18) transformée par (17). Les flèches indiquent les températures de cohérence dans chaque cas.

L'application d'un champ magnétique alternatif $B_{0} \mathrm{e}^{i \omega t}$ selon l'axe d'un échantillon granulaire cylindrique produit une augmentation sensible de la résistance dynamique, surtout à $T<T_{0}$.

L'explication qualitative de ce phénomène est la suivante : d'après la loi de Faraday, le champ induit une tension distribuée sur la périphérie de l'échantillon, proportionnelle à $\omega$ et à $B_{0}$, de même fréquence que le champ. Cette tension détruit la cohérence de phase. En conséquence, toute combinaison de $B_{0}$ et $\omega$ telle que $B_{0} \omega=$ Cte devrait produire, à température fixe, la même variation de résistance dynamique. C'est effectivement le cas de la figure 5. Signalons que ceci nous permet, par une simple mesure de tension, de détecter des champs de l'ordre de $10^{-5} \mathrm{G}$ à $1 \mathrm{MHz}$.

Nous avons aussi commencé l'étude des systèmes bidimensionnels formés par des microponts reliant des couches minces carrées de $50 \mu$ de côté. Chaque échantillon comprend 40000 microponts, dont le détail apparaît sur la figure 6 . Les transitions résistives (Fig. 7) montrent, comme dans le cas tridimensionnel,

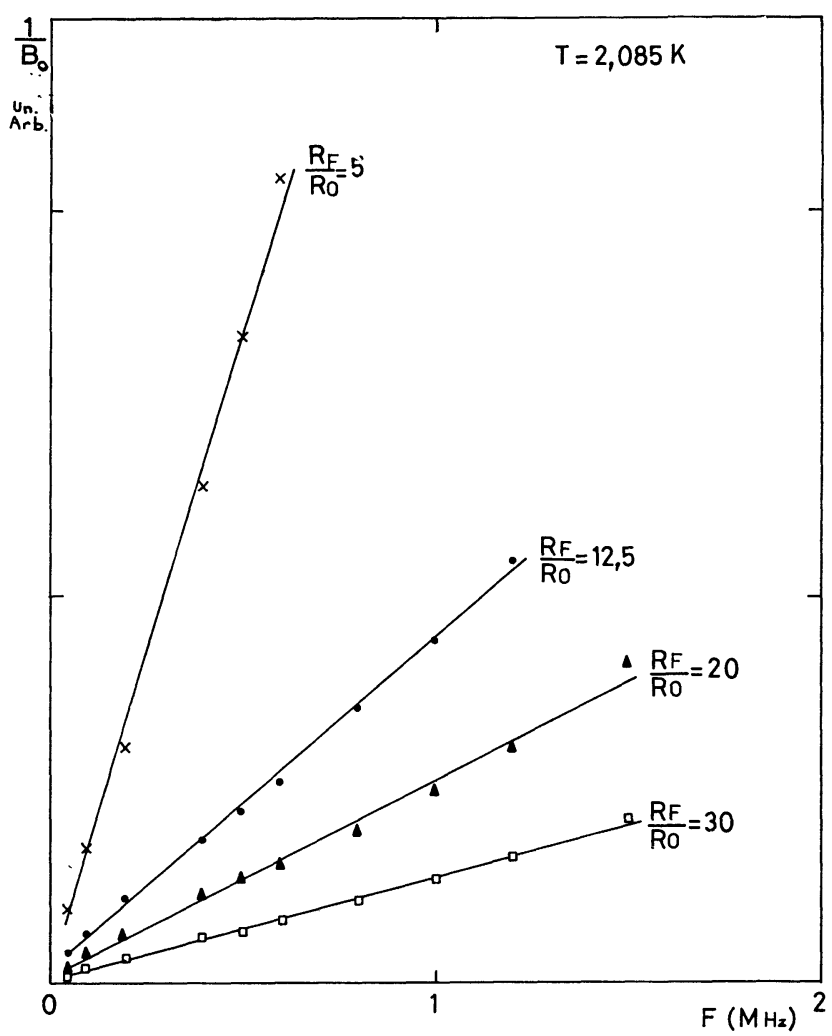

FIG. 5. - Valeurs expérimentales de $B_{0}$ (amplitude du champ magnétique alternatif) appliqué à un supraconducteur granulaire massif de $\mathrm{Nb}$ en fonction de la fréquence $F=\omega / 2 \pi$ produisant des rapports $R_{F} / R_{0}$ donnés. $R_{F}$ est la résistance dynamique en présence du champ et $R_{0}$ est la même quantité en champ nul. La température indiquée est inférieure à $T_{0}$.

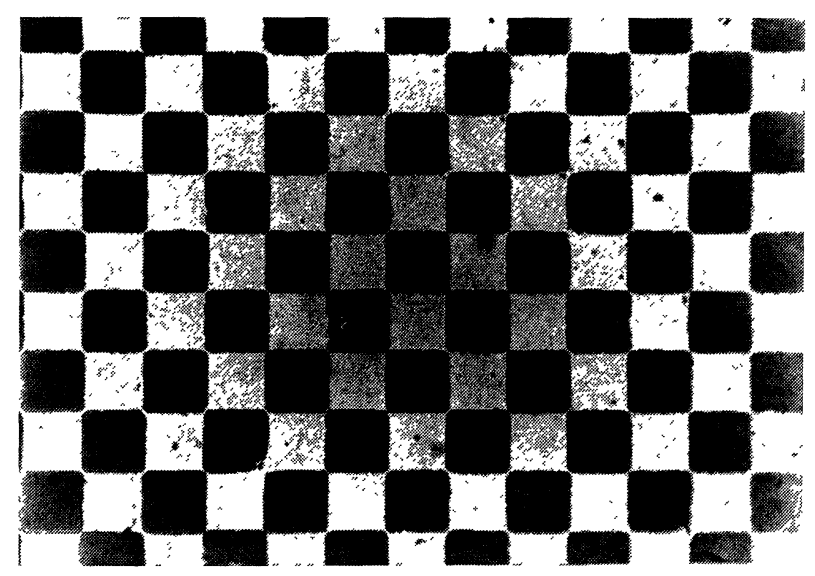

FIG. 6. - Vue partielle d'un échantillon bidimensionnel d'indium comportant 40000 microponts.

un élargissement par rapport aux transitions d'une couche témoin. Mais la résistance commence à diminuer à $T>T_{\mathrm{c}}$, indiquant vraisemblablement l'existence de phénomènes de paraconductivité [11].

4. Caractéristiques de la transition paracohérencecohérence. - Les systèmes que nous avons étudiés présentent des propriétés qui les distinguent nettement des supraconducteurs qui les constituent, en particu- 


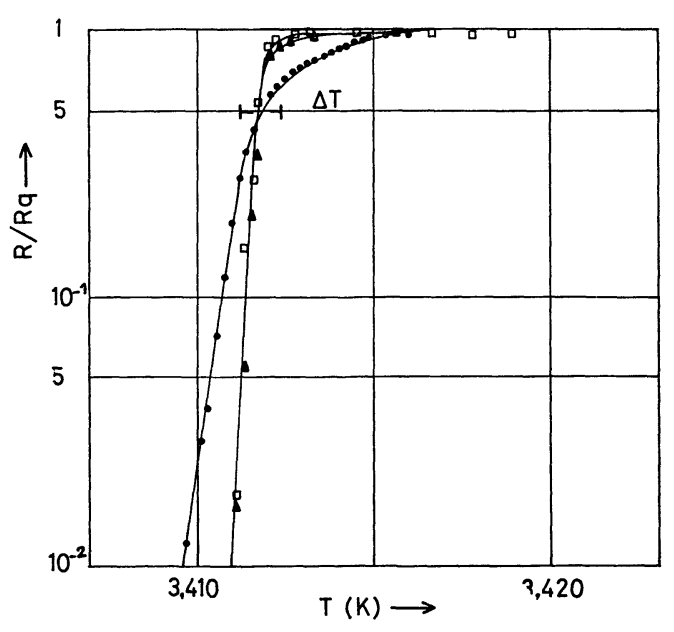

FIg. 7. - Résistance réduite de films d'indium de $2000 \AA$ d'épaisseur en fonction de la température. $\square$ couche témoin évaporée en même temps que les microponts; $\Delta$ ensemble de microponts de $3 \mu$ de largeur; idem $1,5 \mu ; \Delta T$ indique la marge d'erreur sur la détermination de la température.

lier en ce qui concerne les longueurs caractéristiques. On peut estimer, par analogie avec le cas d'une seule jonction, la profondeur de pénétration d'un champ magnétique statique :

$$
\lambda=\frac{\Phi_{0}}{2 \pi}\left[\frac{d}{4 \pi<E>}\right]^{1 / 2} \sim 1-10 \mathrm{~mm},
$$

où $d$ est la distance entre les grains.

Le fait que l'interaction soit limitée aux premiers voisins suggère que la longueur de cohérence est

$$
\xi(0) \simeq d, \quad \xi(T)=d\left[\frac{T_{0}}{T_{0}-T}\right]^{1 / 2}
$$

dans une théorie de champ moléculaire. D'autre part, il est connu que les interactions à très courte portée se traduisent par des transitions de phase de type $\lambda$ (cas de l' $H_{\mathrm{e}}$ ) auxquelles l'hypothèse du champ moléculaire n'est pas applicable. Effectivement, dans le cas des supraconducteurs granulaires massifs, on peut estimer [7] que la région critique de température où les fluctuations sont prédominantes est de l'ordre de $10^{10}$ fois celle des supraconducteurs. Ceci correspond bien à la région « arrondie » de la transition près de $T_{0}$ (Fig. 4), mais il faudrait éliminer toute dispersion dans les propriétés des contacts pour en tirer des conclusions définitives.

\section{Bibliographie}

[1] Josephson, B. D., Adv. Phys. 14 (1965) 419.

[2] Anderson, P. W., Prog. Low Temp. Phys. 5 (1967) 1.

[3] Jaynes, E. T., Phys. Rev. 106 (1957) 620.

[4] Scalapino, D. J., Sears, M. and Ferrell, R. A., Phys. Rev. B 6 (1972) 3409.

[5] Simmonds, M. and Parker, W. H., Phys. Rev. Lett. 24 (1970) 877.

[6] Cortes, H., Pellan, P. and Rosenblatt, J., Proc. 12th Int. Conf. on Low Temp. Phys. (Keigaku Publishing Co., Tokyo) (1970) 487.
[7] Pellan, P., Dousselin, G., Cortes, H. and Rosenblatt, J., Solid State Commun. 11 (1972) 427.

[8] Bethe, H. A., Proc. R. Soc. A 216 (1935) 45.

Domb, C., Adv. Phys. 9 (1960) 149.

[9] Ambegaokar, V. and Baratoff, A., Phys. Rev. Lett. 10 (1963) 486 ; 11 (1963) 104.

[10] Ambegaokar, V. and Halperin, B. I., Phys. Rev. Lett. 22 (1969) 1364.

[11] Ferrel, R."A., J. Low Temp. Phys. 1 (1969) 423. 